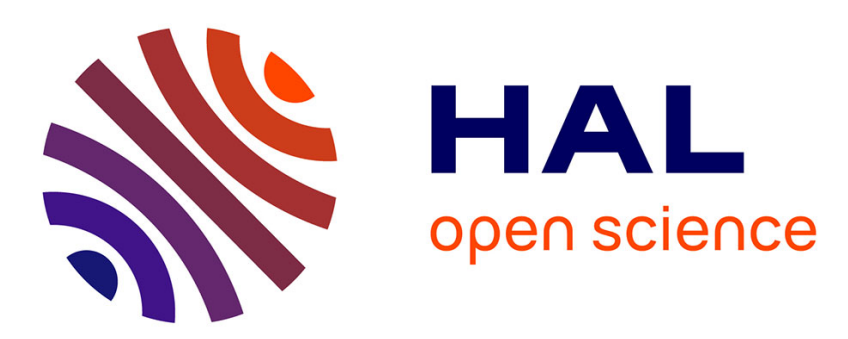

\title{
Chelation and Bridging of Zinc(II) by Tetradentate N2S2 Donor Ligands: Similarities and Differences to Copper(I) and Copper(II) Systems
}

\author{
Wolfgang Kaim
}

\section{- To cite this version:}

Wolfgang Kaim. Chelation and Bridging of Zinc(II) by Tetradentate N2S2 Donor Ligands: Similarities and Differences to Copper(I) and Copper(II) Systems. Journal of Inorganic and General Chemistry / Zeitschrift für anorganische und allgemeine Chemie, 2009, 636 (2), pp.385. 10.1002/zaac.200900373 . hal-00530434

\section{HAL Id: hal-00530434 \\ https://hal.science/hal-00530434}

Submitted on 29 Oct 2010

HAL is a multi-disciplinary open access archive for the deposit and dissemination of scientific research documents, whether they are published or not. The documents may come from teaching and research institutions in France or abroad, or from public or private research centers.
L'archive ouverte pluridisciplinaire HAL, est destinée au dépôt et à la diffusion de documents scientifiques de niveau recherche, publiés ou non, émanant des établissements d'enseignement et de recherche français ou étrangers, des laboratoires publics ou privés. 


\section{Chelation and Bridging of Zinc(II) by Tetradentate N2S2 Donor Ligands: Similarities and Differences to Copper(I) and Copper(II) Systems}

\begin{tabular}{|r|l|}
\hline Journal: & Zeitschrift für Anorganische und Allgemeine Chemie \\
\hline Manuscript ID: & zaac. 200900373 \\
\hline Wiley - Manuscript type: & Article \\
\hline Date Submitted by the \\
Author: & 06-Aug-2009 \\
\hline Complete List of Authors: & $\begin{array}{l}\text { Kaim, Wolfgang; Institute of Inorganic Chemistry, Stuttgart } \\
\text { University }\end{array}$ \\
\hline Keywords: & $\begin{array}{l}\text { Benzimidazole, Crystal structure, Thioether ligands, Zinc } \\
\text { compounds }\end{array}$ \\
\hline
\end{tabular}

\section{(s) ScholaroNE \\ Manuscript Central}




\title{
Chelation and Bridging of Zinc(II) by Tetradentate $\mathrm{N}_{2} \mathrm{~S}_{2}$ Donor Ligands: Similarities and Differences to Copper(I) and Copper(II) Systems
}

\author{
Johannes Schnödt, ${ }^{[\mathrm{a}]}$ Monika Sieger, ${ }^{[\mathrm{a}]}$ Thomas Schleid, ${ }^{[\mathrm{a}]}$ Ingo Hartenbach, ${ }^{[\mathrm{a}]}$ and \\ Wolfgang Kaim*[a]
}

Dedicated to Professor Hans-Jörg Deiseroth on the Occasion of his 65. Birthday

\begin{abstract}
Keywords: Benzimidazole; Crystal structure; Thioether ligands; Zinc compounds
\end{abstract}

\begin{abstract}
Reaction of zinc(II) triflate with neutral bis(benzimidazole-thioether) ligands yielded the complexes $\left[\mathrm{Zn}(\mathrm{bmbdh})\left(\mathrm{H}_{2} \mathrm{O}\right)\left(\eta^{1}-\mathrm{SO}_{3} \mathrm{CF}_{3}\right)\right]\left(\mathrm{SO}_{3} \mathrm{CF}_{3}\right)$ (1) with tetradentate chelating 1,6-bis(N-methylbenzimidazol-2-yl)-2,5-dithiahexane (bmbdh), and dinuclear $\left\{\left[\mathrm{Zn}(\mu \text {-bmdhp })\left(\mathrm{H}_{2} \mathrm{O}\right)\left(\eta^{1}-\mathrm{SO}_{3} \mathrm{CF}_{3}\right)\right]_{2}\right\}\left(\mathrm{SO}_{3} \mathrm{CF}_{3}\right)_{2}$ (2) with bis-bidentate bridging 1,7-bis $(\mathrm{N}$ methylbenzimidazol-2-yl)-2,6-dithiaheptane (bmdhp). In both cases the oxygen donor ligands aqua and triflate adopt cis positions at the approximately octahedrally configurated $\mathrm{Zn}^{2+}$ ions. The hexacoordination is facilitated by rather long $\mathrm{Zn}-\mathrm{S}$ (thioether) distances in contrast to the typically shorter Zn-S(thiolate) bonds from classical zinc finger proteins or their models. With the shorter central $\left(\mathrm{CH}_{2}\right)_{\mathrm{n}}$ chain $(\mathrm{n}=2)$ the ligand bmbdh exhibits facial coordination of the two NSS donor sets in $\mathbf{1}$, similar as in $[\mathrm{Cu}(\mathrm{bmdhp})]^{+}$ions with lower coordinate copper(I) but in contrast to the related $\left[\mathrm{Cu}^{\mathrm{II}}(\mathrm{bmdhp})\left(\mathrm{H}_{2} \mathrm{O}\right)\right]\left(\mathrm{ClO}_{4}\right)_{2}$ with the longer chain ligand bmdhp. In 2, the bis-bidentate coordination of bmdhp ligands to each $\mathrm{Zn}^{2+}$ ion occurs in an all-cis fashion for the pairs of $\mathrm{N}, \mathrm{S}$ and $\mathrm{O}$ donor atoms, in contrast to related $\left[\mathrm{Cu}^{\mathrm{I}}(\mathrm{mmb})_{2}\right]\left(\mathrm{BF}_{4}\right)$, $\mathrm{mmb}=1$-methyl-2-methylthiomethyl-1H-benzimidazole.
\end{abstract}




\author{
*Prof. Dr. W. Kaim \\ E-mail: kaim@iac.uni-stuttgart.de \\ [a] Institut für Anorganische Chemie \\ Universität Stuttgart \\ Pfaffenwaldring 55 \\ 70569 Stuttgart
}

\title{
Introduction
}

In contrast to mainly "hard" or mainly "soft" metal ions the $\mathrm{Zn}^{2+}$ ion can be equally well bonded through $\mathrm{O}, \mathrm{N}$ and $\mathrm{S}$ donor ligands in corresponding proteins [1,2]. Although the coordination has to be compatible with the biological function such as enzymatic catalysis, structural stabilization, or transport, there are many cases where mixed donor ligation from histidine, cysteinate, aspartate or glutamate has been found for zinc in its natural biological environment $[2,3]$. In addition, most hydrolytic enzymes offer at least one "free" coordination site for substrate binding, usually occupied by labile water [1-3].

The $\mathrm{N}_{2} \mathrm{~S}_{2}$ ligation of the zinc ion has received much attention [4-7] since zinc finger proteins were discovered to contain modular motifs ("fingers") involving two neutral histidine- $\mathrm{N}$ and two anionic cysteinato-S donor atoms coordinated to $\mathrm{Zn}^{2+}[1-3,5]$ These zinc fingers (Figure 1) act as structure-maintaining entities for specific protein-nucleic acid recognition in the transcription process [3,5]. In connection with modelling the zinc finger coordination there have been previous synthetic and structural reports on complexes with bis(thiolato)-bis(imidazole) [6] or -bis(benzimidazole) [7] and bis(thioether)-bis(pyridyl) or -bis(amine) coordinated zinc, with and without additional $\mathrm{O}$ donor ligation [8]. Bis(thioether)-bis(benzimidazole) ligation has not yet been described. 


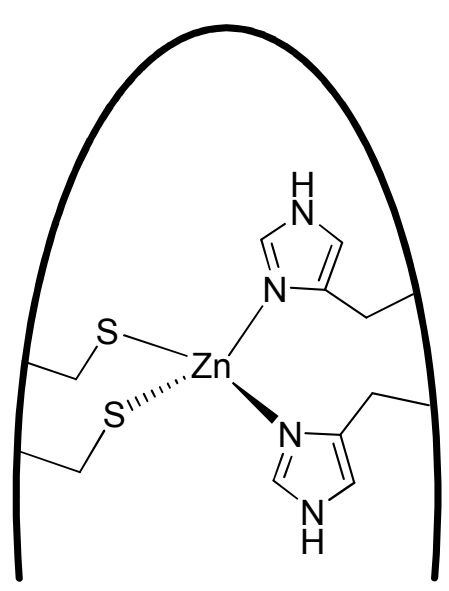

Figure 1. Zinc finger.

Probing the mixed benzimidazole-thioether ligand binding to copper(I) and copper(II) [9] with a view on $\mathrm{O}_{2}$ utilising monooxygenases such as PHM ( $\alpha$-peptidylglycine hydroxylating monooxygenase) with its $\mathrm{Cu}(\mathrm{His})_{2}(\mathrm{Met})$ arrangement $(\mathrm{His}=$ histidine, Met $=$ methionine) [10] we have been interested in the coordination characteristics of mixed $\mathrm{N}_{2} \mathrm{~S}_{2}$ donor ligands involving neutral S(thioether) functions in the absence of special effects such as Jahn-Teller distortion $\left(\mathrm{Cu}^{\mathrm{II}}[11]\right)$ or low coordination number preference $\left(\mathrm{Cu}^{\mathrm{I}}\right)$ [12]. We therefore report here our results from the reactions of zinc triflate with the tetradentate bis(benzimidazole-thioether) ligands $\mathrm{bmbdh}=1,6$-bis(N-methylbenzimidazol-2-yl)-2,5dithiahexane and bmdhp = 1,7-bis(N-methylbenzimidazol-2-yl)-2,6-dithiaheptane (see Scheme 1) which have already been used to obtain partially structurally characterized complexes such as $\left[\mathrm{Cu}(\mathrm{bmdhp})\left(\mathrm{H}_{2} \mathrm{O}\right)\right]\left(\mathrm{ClO}_{4}\right)_{2}$ and $[\mathrm{Cu}(\mathrm{bmdhp})](\mathrm{X}), \mathrm{X}=\mathrm{ClO}_{4}, \mathrm{O}_{3} \mathrm{SCF}_{3}$ $[11,13]$<smiles>Cn1c(CSCCSCc2nc3ccccc3n2C)nc2ccccc21</smiles><smiles>Cn1c(CSCCCSCc2nc3ccccc3n2C)nc2cc[c][c]c21</smiles>

\section{Scheme 1.}




\section{Results and Discussion}

Reaction of zinc triflate in acetone with the neutral bis(benzimidazole-thioether) ligands from Scheme 1 yielded two different kinds of compounds. In the absence of electron or charge transfer activity and lacking prominent signature features in IR or NMR spectroscopy the characterization of the two seemingly $1: 1$ complexes $\left[\mathrm{Zn}(\mathrm{L})\left(\mathrm{H}_{2} \mathrm{O}\right)(\mathrm{OTf})_{2}\right]$ obtained from $\mathrm{L}$ $=$ bmbdh or bmdhp and $\mathrm{Zn}(\mathrm{OTf})_{2}$ had to rely on the molecular structure analysis of single crystalline material. Crystallographic information and bond parameters are listed in Tables 1 and 2, Figures 2 and 3 show the molecules in their setting.

Tabelle 1. Crystallographic and structure refinement data.

\begin{tabular}{|c|c|c|}
\hline & $\mathbf{1} * 2 \mathrm{OC}\left(\mathrm{CH}_{3}\right)_{2}$ & $2 * 2 \mathrm{OC}\left(\mathrm{CH}_{3}\right)_{2}$ \\
\hline Formula & $\mathrm{C}_{28} \mathrm{H}_{36} \mathrm{~F}_{6} \mathrm{~N}_{4} \mathrm{O}_{9} \mathrm{~S}_{4} \mathrm{Zn}$ & $\mathrm{C}_{29} \mathrm{H}_{38} \mathrm{~F}_{6} \mathrm{~N}_{4} \mathrm{O}_{9} \mathrm{~S}_{4} \mathrm{Zn}$ \\
\hline Formula weight $/ \mathrm{gmol}^{-1}$ & 880.22 & 894.24 \\
\hline Crystal system & triclinic & triclinic \\
\hline Space group & P-1 & P-1 \\
\hline Cell parameters a /Å & $10.902(0)$ & $9.517(0)$ \\
\hline $\mathrm{b} / \AA$ & $12.109(1)$ & $13.317(1)$ \\
\hline $\mathrm{c} / \AA$ & $15.348(1)$ & $15.554(1)$ \\
\hline$\alpha /{ }^{\circ}$ & $71.244(2)$ & $87.543(3)$ \\
\hline$\beta /^{\circ}$ & $88.773(2)$ & $86.136(3)$ \\
\hline$\gamma /{ }^{\circ}$ & $71.129(2)$ & $73.284(3)$ \\
\hline $\mathrm{V} / \AA^{3}$ & $1807.72(13)$ & $1883.10(15)$ \\
\hline $\mathrm{Z}$ & 2 & 2 \\
\hline
\end{tabular}


1

3
Calc. density $/ \mathrm{g} \mathrm{cm}^{-3}$

Temperature /K

$\theta$ Range ${ }^{\circ}$

$\mathrm{F}(000)$

Index range

Reflns. collect.

Reflns. unique

Reflns. gt

$\mu / \mathrm{mm}^{-1}$

Parameters

Goodness of fit

$\mathrm{R}_{1} / \mathrm{wR}_{2}$ [all]

$\mathrm{R}_{1} / \mathrm{wR}_{2}[\mathrm{I}>2 \sigma(\mathrm{I})]$

$\Delta \rho / \AA^{-3}$
1.617

100(1)

$0.41-27.48$

904

$-14<h>12 ;-15<k>15$;

$-19<l>19$

numerical

33943

8242

5214

0.997

511

1.006

$0.1135 / 0.1512$

0.0594 / 0.1273

$0.588 /-0.655$
1.577

100(1)

$0.41-23.26$

920

$-10<h>10 ;-14<k>14$;

$-17<l>17$

numerical

29275

5351

3884

0.959

522

1.108

0.0988 / 0.1399

$0.0588 / 0.1184$

$0.704 /-0.759$ 
Tabelle 2. Selected bond lengths and bond angles.

\begin{tabular}{|c|c|c|}
\hline & $\mathbf{1} * 2 \mathrm{OC}\left(\mathrm{CH}_{3}\right)_{2}$ & $2 * 2 \mathrm{OC}\left(\mathrm{CH}_{3}\right)_{2}$ \\
\hline $\mathrm{Zn}-\mathrm{O} 1$ & $2.051(3) \AA$ & $2.089(5) \AA$ \\
\hline $\mathrm{Zn}-\mathrm{O} 2$ & $2.134(3) \AA$ & $2.151(4) \AA$ \\
\hline $\mathrm{Zn}-\mathrm{S} 1$ & $2.595(1) \AA$ & $2.665(2) \AA$ \\
\hline $\mathrm{Zn}-\mathrm{S} 2$ & $2.625(1) \AA$ & 2.591(2) $\AA$ \\
\hline Zn-N1 & 2.097(4) $\AA$ & $2.048(5) \AA$ \\
\hline $\mathrm{Zn}-\mathrm{N} 3$ & 2.094(4) $\AA$ & $2.076(5) \AA$ \\
\hline $\mathrm{O} 1-\mathrm{Zn}-\mathrm{S} 2$ & $175.71(10)^{\circ}$ & $80.02(14)^{\circ}$ \\
\hline $\mathrm{O} 1-\mathrm{Zn}-\mathrm{N} 3$ & $96.12(14)^{\circ}$ & $91.1(2)^{\circ}$ \\
\hline $\mathrm{O} 1-\mathrm{Zn}-\mathrm{N} 1$ & $90.39(14)^{\circ}$ & $162.2(2)^{\circ}$ \\
\hline $\mathrm{O} 1-\mathrm{Zn}-\mathrm{O} 2$ & $89.43(12)^{\circ}$ & $90.39(18)^{\circ}$ \\
\hline $\mathrm{O} 1-\mathrm{Zn}-\mathrm{S} 1$ & $97.40(9)^{\circ}$ & $83.66(15)^{\circ}$ \\
\hline N3-Zn-N1 & $167.28(13)^{\circ}$ & $105.8(2)^{\circ}$ \\
\hline $\mathrm{N} 3-\mathrm{Zn}-\mathrm{O} 2$ & $96.43(12)^{\circ}$ & $93.31(18)^{\circ}$ \\
\hline N3-Zn-S2 & $79.98(10)^{\circ}$ & $79.74(15)^{\circ}$ \\
\hline N3-Zn-S1 & $89.09(9)^{\circ}$ & $172.83(15)^{\circ}$ \\
\hline $\mathrm{S} 1-\mathrm{Zn}-\mathrm{O} 2$ & $170.74(9)^{\circ}$ & $91.63(12)^{\circ}$ \\
\hline $\mathrm{S} 1-\mathrm{Zn}-\mathrm{S} 2$ & $84.38(4)^{\circ}$ & $94.48(5)^{\circ}$ \\
\hline S1-Zn-N1 & $79.21(9)^{\circ}$ & $78.95(15)^{\circ}$ \\
\hline
\end{tabular}


$97.35(15)^{\circ}$

N1-Zn-O2

$94.56(12)^{\circ}$

$93.98(18)^{\circ}$

S2-Zn-O2

$89.24(8)^{\circ}$

$167.97(12)^{\circ}$

C9-S1-Zn $\quad 98.92(14)^{\circ} \quad 95.8(2)^{\circ}$

C9-S1-C10 $\quad 101.6(2)^{\circ} \quad 100.1(3)^{\circ}$

C10-S1-Zn $\quad 100.18(15)^{\circ} \quad 101.3(2)^{\circ}$

$\begin{array}{lll}\mathrm{C} 20-\mathrm{S} 2-\mathrm{Zn} & 100.34(15)^{\circ} & 108.5(2)^{\circ}\end{array}$

$\begin{array}{lll}\mathrm{C} 20-\mathrm{S} 2-\mathrm{C} 19 \quad 100.7(2)^{\circ} & 101.6(3)^{\circ}\end{array}$

$\mathrm{C} 19-\mathrm{S} 2-\mathrm{Zn} \quad 97.50(15)^{\circ} \quad 94.4(2)^{\circ}$

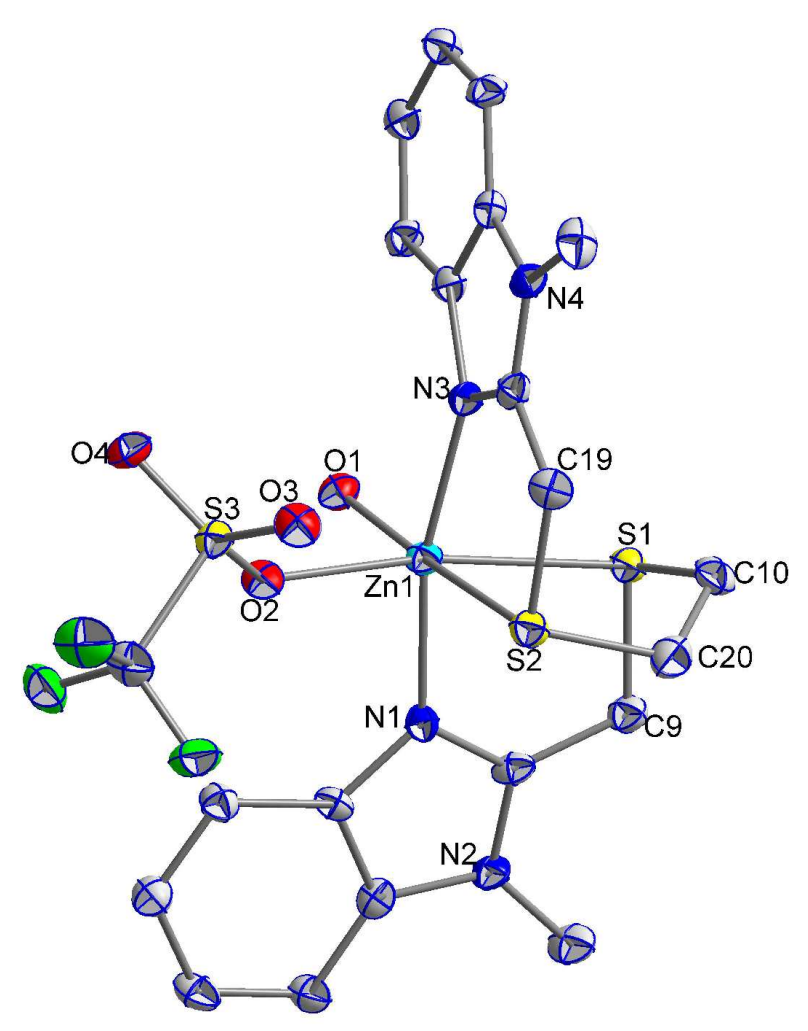

Figure 2. Molecular structure of $\left[\mathrm{Zn}(\mathrm{bmbdh})\left(\mathrm{H}_{2} \mathrm{O}\right)\left(\eta^{1}-\mathrm{SO}_{3} \mathrm{CF}_{3}\right)\right]\left(\mathrm{SO}_{3} \mathrm{CF}_{3}\right)(\mathbf{1})$ in the crystal (acetone molecules are not shown). 


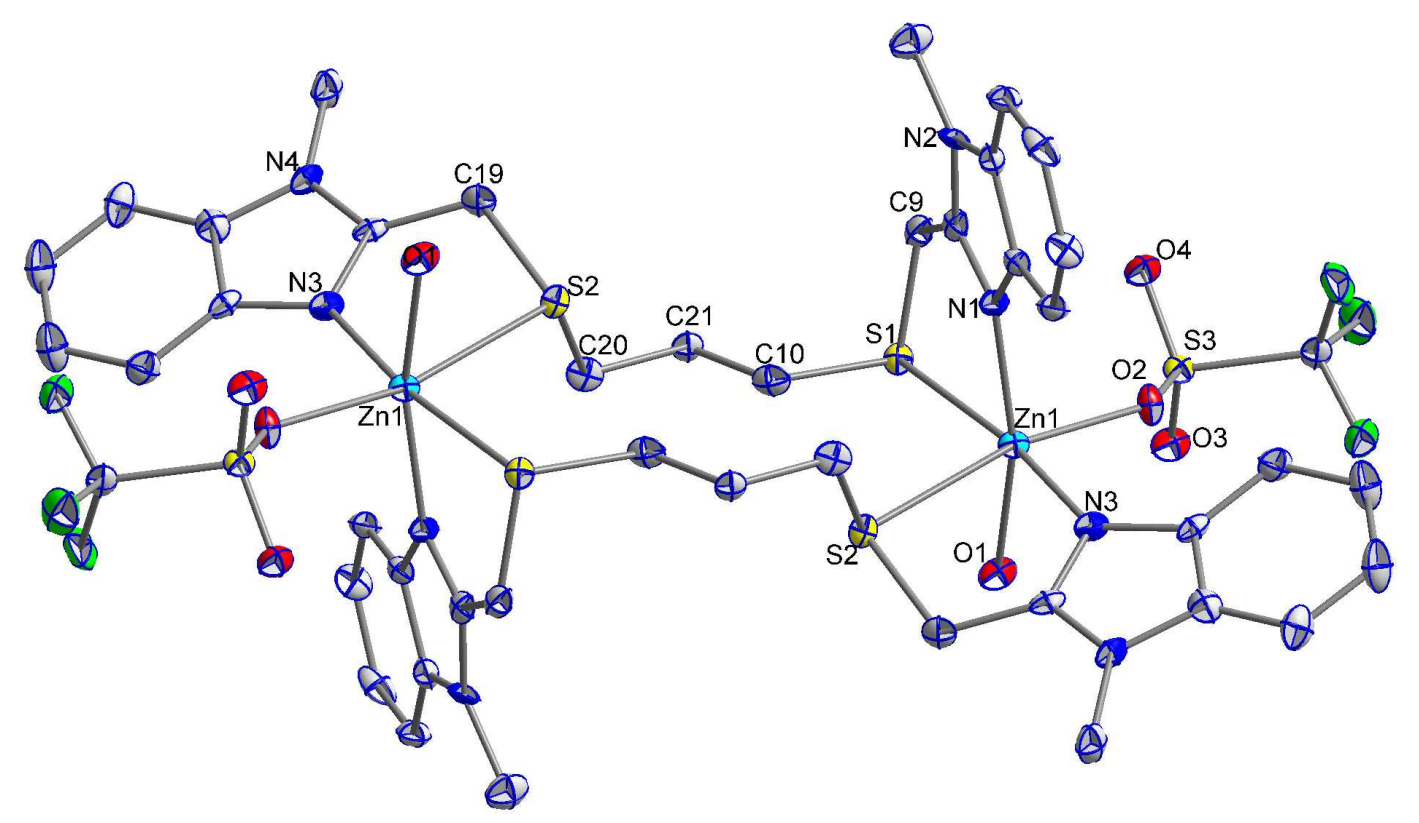

Figure 3. Molecular structure of $\left\{\left[\mathrm{Zn}(\mu-\mathrm{bmdhp})\left(\mathrm{H}_{2} \mathrm{O}\right)\left(\eta^{1}-\mathrm{SO}_{3} \mathrm{CF}_{3}\right)\right]_{2}\right\}\left(\mathrm{SO}_{3} \mathrm{CF}_{3}\right)_{2}(2)$ in the crystal (acetone molecules are not shown).

In both cases $\mathbf{1}$ and $\mathbf{2}$ the oxygen donor ligands aqua and triflate adopt cis positions at the approximately octahedrally configurated $\mathrm{Zn}^{2+}$ ions. This coordination number is tolerated because of the presence of four light donor atoms $(2 \mathrm{O}, 2 \mathrm{~N})$ and two weakly bonded $\mathrm{S}$ (thioether) donor centers. Specifically, the rather long Zn-S(thioether) distances at about $2.60 \AA$ stand in contrast to the typically shorter $\mathrm{Zn}-\mathrm{S}$ (thiolate) bonds (ca. $2.3 \AA$ ) from classical zinc finger proteins $[3,5]$ or their models $[4,6,7]$.

Remarkably, the two compounds $\mathbf{1}$ and $\mathbf{2}$ showed a different degree of aggregation. Compound 1 obtained with bmbdh turned out to be a mononuclear complex with one aqua and one triflate ligand coordinated in cis position of $\mathrm{Zn}^{2+}$ in $\left[\mathrm{Zn}(\mathrm{bmbdh})\left(\mathrm{H}_{2} \mathrm{O}\right)\left(\eta^{1}-\right.\right.$ $\left.\left.\mathrm{SO}_{3} \mathrm{CF}_{3}\right)\right]\left(\mathrm{SO}_{3} \mathrm{CF}_{3}\right.$ ) (Figure 2,4). The tetradentate chelating bmbdh ligand adopts an arrangement with cis positioned thioether-S donors and trans positioned imine-N donor atoms from the benzimidazoles (fac/fac combination of NSS and SSN). At $167.3(1)^{\circ}$ the N-Zn-N angle is fairly close to linearity. 

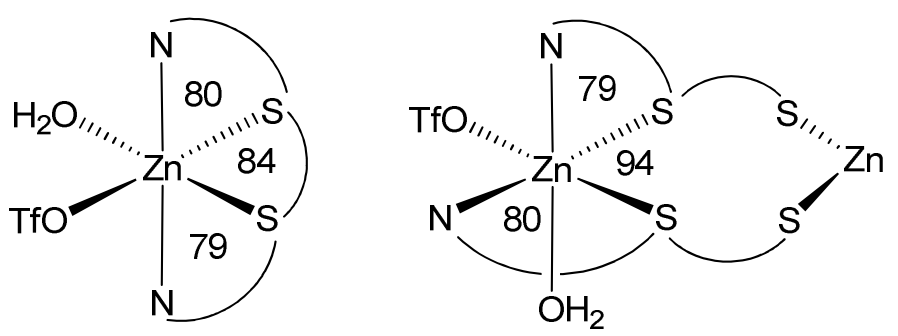

Figure 4. Coordination arrangements for $\mathbf{1}$ (left) and $\mathbf{2}$ (right) (bond angles in deg.).

On the other hand, bmdhp in compound 2 , $\left\{\left[\mathrm{Zn}(\mu \text {-bmdhp })\left(\mathrm{H}_{2} \mathrm{O}\right)\left(\eta^{1}-\mathrm{SO}_{3} \mathrm{CF}_{3}\right)\right]_{2}\right\}\left(\mathrm{SO}_{3} \mathrm{CF}_{3}\right)_{2}$, acts as a bis(bidentate) bridge between two zinc ions (Figures 3,4). Again, the aqua ligand (probably from less than $100 \%$ dry acetone) and one ${ }^{-}$OTf anion occupy a cis position at the approximately octahedrally configurated metal, however, this time the two independent N,S chelates from two bmdhp molecules bond with the two $\mathrm{N}$ atoms also in cis position (angle $\left.\mathrm{N}-\mathrm{Zn}-\mathrm{N} 105.8(2)^{\circ}\right)$, like the two S donors (allcis situation).

The configuration of mononuclear $\mathbf{1}$ is somewhat reminiscent of that of $\left[\mathrm{Cu}(\mathrm{mmb})_{2}\right]^{+}$ [9b], mmb = 1-methyl-2-methylthiomethyl-1H-benzimidazole, which involves the $3 \mathrm{~d}^{10}$ configuration $\mathrm{Cu}^{+}\left(\right.$like $\mathrm{Zn}^{2+}$ ) and exhibits a large $\mathrm{N}-\mathrm{Cu}-\mathrm{N}$ angle of $169.8(1)^{\circ}$ (Figure 5, bottom left) but leaves a "free" sector where the $\mathrm{Zn}$ complex bonds two O donor ligands. The configuration in compound $\mathbf{2}$ has no such precedent in copper chemistry because the structural preference of $\mathrm{Cu}^{\mathrm{I}}$ is for smaller coordination numbers (e.g. 2, 2+2, 4) as evident for $\left[\mathrm{Cu}(\mathrm{mmb})_{2}\right]^{+}[9 \mathrm{~b}]$ and $[\mathrm{Cu}(\mathrm{bmdhp})]^{+}$(angle $\mathrm{N}-\mathrm{Cu}-\mathrm{N}$ of ca. $151^{\circ}$ [13], Figure 5 , top left). The structures of copper(II) analogues such as $\left[\mathrm{Cu}(\mathrm{bmdhp})_{2}\left(\mathrm{OH}_{2}\right)\right][11]$ or $\left[\mathrm{Cu}(\mathrm{mmb})_{2}\left(\mathrm{OClO}_{3}\right)\right]^{+}$ [14] are dominated by the Jahn-Teller effect which leaves a planar mer/mer arrangement for the N,S,S,N donor set at pentacoordinate $\mathrm{Cu}^{2+}$ (Figure 5, top and bottom right). 
<smiles>CSCc1nc2ccccc2n1C</smiles>

Scheme 2.
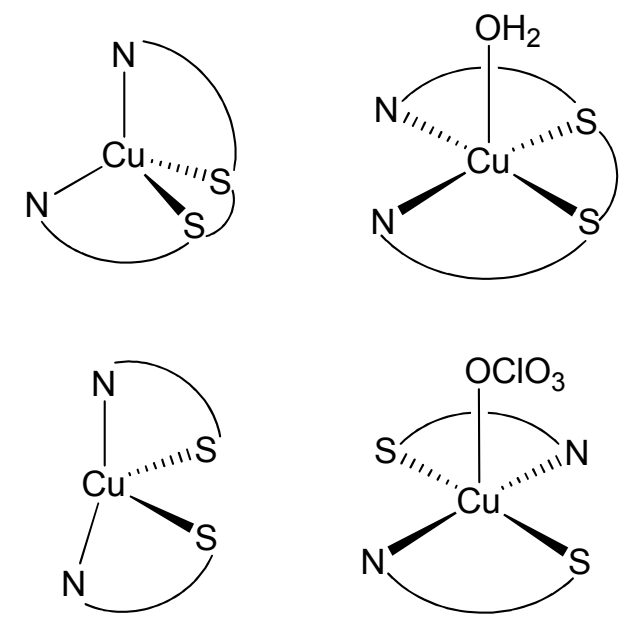

Figure 5. Coordination arrangements in copper(I) (left) and copper(II) complexes (right) of bmdhp (top) and mmb (bottom) (from refs. 9b,11b,13,14).

The absence of special ligand-field or low-coordination-number effects as in copper(II) or copper(I) compounds leaves the $\mathrm{Zn}^{2+}$ ion in a seemingly "normal" octahedral configuration which, however, still displays considerable structural variability. The weak bonding of the thioether donors at about $2.6 \AA \mathrm{Zn}$-S distance allows for enough space to adopt additional $\mathrm{O}$ donors which is less likely when strongly coordinated thiolate ligands such as in zinc finger configurations and their models are present $\left(\mathrm{d}_{\mathrm{Zn}-\mathrm{S}}\right.$ ca. $2.3 \AA$ [4-7]). Then, the tetracoordinate arrangement is the alternative which may also offer a greater degree of structural (angular) flexibility than the hexacoordinate situation.

Using a tetradentate $\mathrm{N}_{2} \mathrm{~S}_{2}$ donor ligand type which has already been established in copper coordination chemistry $[9,11]$ and which offers biomimetic benzimidazole (-> 
histidine) as well as thioether (-> methionine) coordination functions, we have obtained two different kinds of complexes, depending on the length of the oligomethylene spacer between the two molecular halves of the ligand. In both cases, however, a hexacoordination is observed with two additional $\mathrm{O}$ donors (counterion, water) and the weakly bonded S(thioether) donors in the cis position. Clearly this coordination arrangement differs from that of zinc fingers and related proteins [5]. Nevertheless, chelate binding of zinc(II) under at least partially physiological conditions is an attractive option which may find uses in the increasingly investigated [15] biochemistry and medicinal chemistry.

\section{Experimental Section}

\section{General Information}

Commercially available acetone (Merck) was dried by stirring for $10 \mathrm{~d}$ with $\mathrm{CaSO}_{4}$ and subsequent distillation. $\mathrm{Zn}\left(\mathrm{SO}_{3} \mathrm{CF}_{3}\right)_{2}$ from Sigma Aldrich was used without further purification. All reactions involving metal complexes were conducted under argon using standard Schlenk technique.

\section{Instrumentation}

${ }^{1} \mathrm{H}-\mathrm{NMR}$ spectra were taken on a Bruker AC 250 spectrometer.

$\left[\mathrm{Zn}(\mathrm{bmbdh})\left(\mathrm{H}_{2} \mathrm{O}\right)\left(\eta^{l}-\mathrm{SO}_{3} \mathrm{CF}_{3}\right)\right]\left(\mathrm{SO}_{3} \mathrm{CF}_{3}\right)(\mathbf{1})$

A suspension of bmbdh [11a] $(69 \mathrm{mg}, 0.181 \mathrm{mmol})$ in $10 \mathrm{ml}$ acetone was added to hygroscopic $\mathrm{Zn}(\mathrm{OTf})_{2}(66 \mathrm{mg}, 0.181 \mathrm{mmol})$ in $15 \mathrm{ml}$ acetone. The product was precipitated by reducing the solvent after stirring for 4 hours at room temperature. A white powder remained after the solvent was decanted. Yield: $128 \mathrm{mg}(0.172 \mathrm{mmol}, 95 \%)$. Elementary analysis: $\mathrm{C}_{22} \mathrm{H}_{24} \mathrm{~F}_{6} \mathrm{~N}_{4} \mathrm{O}_{7} \mathrm{~S}_{4} \mathrm{Zn}\left(764.11\right.$ gmol $^{-1}$ ). C 34.69 (cal. 34.58); H 3.13 (calc. 3.17); N 7.33 (7.33)\%. ${ }^{1} \mathrm{H}-\mathrm{NMR}\left(250 \mathrm{MHz}\right.$, acetone- $\left.D_{6}, 25^{\circ} \mathrm{C}\right): 8.33\left(\mathrm{~d}, \mathrm{~J}^{3}{ }_{\mathrm{H}, \mathrm{H}}=7.5 \mathrm{~Hz}, 2 \mathrm{H}, \mathrm{H}^{4,4^{\prime}}\right), 7.68(\mathrm{~d}$, 


$$
\begin{aligned}
& \left.\mathrm{J}_{\mathrm{H}, \mathrm{H}}^{3}=7.3 \mathrm{~Hz}, 2 \mathrm{H}, \mathrm{H}^{7}, 7^{\prime}\right), 7.43\left(\mathrm{q}, \mathrm{J}_{\mathrm{H}, \mathrm{H}}^{3}=7.2 \mathrm{~Hz}, 4 \mathrm{H}, \mathrm{H}^{5}, 5^{\prime}, 6,6\right), 4.56(\mathrm{~s}, 4 \mathrm{H}, \mathrm{S}- \\
& \mathrm{CH}_{2} \text { (benzimidazole)), } 3.98 \text { (s, 6H, } \mathrm{CH}_{3} \text { ), } 3.13 \text { (s, 4H, S- } \mathrm{CH}_{2} \text { ). }
\end{aligned}
$$

$\left\{\left[\mathrm{Zn}(\mu-b m d h p)\left(\mathrm{H}_{2} \mathrm{O}\right)\left(\eta^{l}-\mathrm{SO}_{3} \mathrm{CF}_{3}\right)\right]_{2}\right\}\left(\mathrm{SO}_{3} \mathrm{CF}_{3}\right)_{2}(2) *\left(\mathrm{CH}_{3}\right)_{2} \mathrm{CO}$

A solution of bmdhp [11b] (91 mg, $0.230 \mathrm{mmol})$ in acetone $(3 \mathrm{ml})$ was added to $\mathrm{Zn}(\mathrm{OTf})_{2}$ (84 $\mathrm{mg}, 0.230 \mathrm{mmol}$ ) dissolved in $15 \mathrm{ml}$ degassed acetone. The product precipitated out of the solution as mono-solvate by reducing the solvent after stirring for 30 minutes at room temperature. Yield: $148 \mathrm{mg}(0.191 \mathrm{mmol}, 83 \%)$. Elementary analysis: $\mathrm{C}_{49} \mathrm{H}_{58} \mathrm{~F}_{12} \mathrm{~N}_{8} \mathrm{O}_{15} \mathrm{~S}_{8} \mathrm{Zn}_{2}$ (1614.35 gmol $^{-1}$ ). C 37.49 (cal. 36.46); H 3.24 (cal. 3.62); N 7.48 (6.94)\%. ${ }^{1} \mathrm{H}-\mathrm{NMR}$ (250 $\mathrm{MHz}$, acetone- $\left.D_{6}, 25^{\circ} \mathrm{C}\right): 7.85\left(\mathrm{~m}, 4 \mathrm{H}, \mathrm{H}^{4,4^{\prime}, 7,7^{\prime}}\right), 7.50\left(\mathrm{t}, \mathrm{J}_{\mathrm{H}, \mathrm{H}}^{3}=7.8 \mathrm{~Hz}, 2 \mathrm{H}, \mathrm{H}^{5,5^{\prime}}\right), 7.35(\mathrm{t}$, $\left.\mathrm{J}_{\mathrm{H}, \mathrm{H}}^{3}=7.7 \mathrm{~Hz}, 2 \mathrm{H}, \mathrm{H}^{6,6^{\prime}}\right), 4.66\left(\mathrm{~s}, 4 \mathrm{H}, \mathrm{S}-\mathrm{CH}_{2}\right.$ (benzimidazole) $), 4.11\left(\mathrm{~s}, 6 \mathrm{H}, \mathrm{CH}_{3}\right), 3.06(\mathrm{~m}$, 4H, S-CH 2$), 1.99\left(\mathrm{~m}, 2 \mathrm{H}, \mathrm{CH}_{2}\right)$.

\section{Crystallography}

Single crystals of $\mathbf{1}$ and $\mathbf{2}$ were grown as colourless needles from acetone solutions at $-10^{\circ} \mathrm{C}$. The X-ray data were collected on a Bruker-Nonius $\kappa$-CCD diffractometer, using graphite monochromated Mo-K $\alpha$ radiation $(\lambda=0.71073 \AA)$. The structures were solved by the Patterson method using the SHELXTL package [15] while refining was carried out with SHELXL97 employing full matrix least-squares methods on $F 2$ with $F \sigma^{2} \geq-2 \sigma\left(F \sigma^{2}\right)$. All nonhydrogen atoms were refined anisotropically, hydrogen atoms were placed in the calculated positions and were refined using the riding model.

Crystallographic data have been deposited with the Cambridge Crystallographic Data Centre, CCDC-743048 - CCDC-743049. Copies of the information may be obtained free of charge from: The Director, CCDC, 12 Union Road, Cambridge CB2 1EZ, UK (fax: (+44) 1223-336Wiley-vch 
033; e-mail: deposit@ccdc.cam.ac.uk; www: http://www.ccdc.cam.ac.uk).

\section{Acknowledgements.}

This work was supported by the Deutsche Forschungsgemeinschaft (SFB 706) and the Fonds der Chemischen Industrie.

\section{References}

[1] W. Kaim, B. Schwederski, Bioinorganic Chemistry: Inorganic Elements in the Chemistry of Life, Wiley, Chichester, 1994. b) W. Kaim, B. Schwederski, Bioanorganische Chemie, 4. Aufl., Teubner, Wiesbaden, 2005.

[2] R. J. P. Williams, J. J. R. Frausto da Silva, The Natural Selection of the Chemical Elements, Oxford University Press, Oxford, 1996.

[3] a) A. Klug, in Zinc Finger Proteins: From Atomic Contact to Cellular Function, S. Iuchi, N. Kuldell (eds), Landes Bioscience and Kluwer, Georgetown and Dordrecht 2005, p.1. b) M. Dhanasekaran, S. Negi, Y. Sugiura, Acc. Chem. Res. 2005, 39, 45. c) O. Vázquez, M. E. Vázquez, J. B. Blanco, L. Castedo, J. L. Mascareñas, Angew. Chem. 2007, 119, 7010; Angew. Chem. Int. Ed. 2007, 46, 6886.

[4] J. Otto, I. Jolk, T. Viland, R. Wonnemann, B. Krebs, Inorg. Chim. Acta 1999, 285, 262.

[5] M. J. Lachenmann, J. E. Ladbury, J. Dong, K. Huang, P. Carey, M. A. Weiss, Biochemistry 2004, 43, 13910.

[6] a) D. T. Corwin, S. A. Koch, Inorg. Chem. 2002, 27, 493. b) B. Müller, A. Schneider, M. Tesmer, H. Vahrenkamp, Inorg. Chem. 1999, 38, 1900. c) M. Bochmann, G. C. Bwembya, R. Grinter, A. K. Powell, K. J. Webb, M. B. Hursthouse, K. M. A. Malik, M. A. Mazid, Inorg. Chem. 2002, 33, 2290. d) W.-Y. Sun, L. Zhang, K.-B. Yu, J. Chem. Soc., Dalton Trans. 1999, 795 
[7] a) R. Burth, H. Vahrenkamp, Inorg. Chim. Acta 1998, 282, 193. b) R. Burth, M. Gelinsky, H. Vahrenkamp, Inorg. Chem. 1998, 37, 2833. c) R. E. Marsh, Acta Crystallogr. Sect. B: Struct. Sci. 2002, 58, 893.

[8] a) D. C. Bebout, W. Lai, S. M. Stamps, S. M. Berry, R. J. Butcher, Polyhedron 2008, 27, 1591. b) P. Chakraborty, S. Kumar Chandra, A. Chakravorty, Inorg. Chim. Acta 1995, 229, 477. c) D. K. Garner, R. A. Allred, K. J. Tubbs, A. M. Arif, L. M. Berreau, Inorg. Chem. 2002, 41, 3533. d) M. M. Makowska-Grzyska, P. C. Jeppson, R. A. Allred, A. M. Arif, L. M. Berreau, Inorg. Chem. 2002, 41, 4872. e) M. Seitz, S. Stempfhuber, M. Zabel, M. Schütz, O. Reiser, Angew. Chem. 2005, 117, 246; Angew. Chem. Int. Ed. 2005, 44, 242.

[9] a) J. Rall, M. Wanner, M. Albrecht, F. M. Hornung, W. Kaim, Chem. Eur. J. 1999, 5, 2802. b) M. Albrecht, K. Hübler, S. Zalis, W. Kaim, Inorg. Chem. 2000, 39, 4731.

[10] S. T. Prigge, B. A. Eipper, R. E. Mains, L. M. Amzel, Science 2004, 304, 864.

[11] a) P. L. Verheijdt, J. G. Haasnoot, J. Reedijk, Inorg. Chim. Acta, 1983, 16, L43. b) A. W. Addison, T. N. Rao, J. Reedijk, J. van Rijn, G. C. Verschoor, J. Chem. Soc. Dalton Trans., 1984, 1349.

[12] a) W. Kaim, J. Rall, Angew. Chem. 1996, 108, 47; Angew. Chem. Int. Ed. Engl. 1996, 35, 43. b) W. Kaim, Dalton Trans. 2003, 761.

[13] J. Schnödt, M. Sieger, B. Sarkar, J. Fiedler, C. Y. Su, W. Kaim, unpublished work.

[14] M. Albrecht, K. Hübler, T. Scheiring, W. Kaim, Inorg. Chim. Acta 1999, 287, 204.

[15] A. I. Anzellotti, N. P. Farrell, Chem. Soc. Rev. 2008, 37, 1629.

[16] G. M. Sheldrick, SHELXL-97, Universität Göttingen, 1997. 